\title{
Understanding the development of flexibility in struggling algebra students
}

\section{Citation}

Newton, Kristie J., Jon R. Star, and Kathleen Lynch. 2010. Understanding the development of flexibility in struggling algebra students. Mathematical Thinking \& Learning, 12, no. 4: 282-305.

\section{Published Version}

doi:10.1080/10986065.2010.482150

\section{Permanent link}

http://nrs.harvard.edu/urn-3:HUL.InstRepos:4889490

\section{Terms of Use}

This article was downloaded from Harvard University's DASH repository, and is made available under the terms and conditions applicable to Open Access Policy Articles, as set forth at http:// nrs.harvard.edu/urn-3:HUL.InstRepos:dash.current.terms-of-use\#OAP

\section{Share Your Story}

The Harvard community has made this article openly available.

Please share how this access benefits you. Submit a story.

Accessibility 
Running head: DEVELOPMENT OF FLEXIBILITY

Exploring the Development of Flexibility in Struggling Algebra Students 


\begin{abstract}
The ability to flexibly solve problems is considered an important outcome for school mathematics and is the focus of this paper. The paper describes the impact of a three-week summer course for students who struggle with algebra. During the course, students regularly compared and contrasted worked examples of algebra problems in order to promote flexible use of solution strategies. Assessments were designed to capture both knowledge and use of multiple strategies. The students were interviewed in order to understand their rationales for choosing particular strategies, as well as their attitudes toward instruction that emphasized multiple strategies. Findings suggest that students gained both knowledge of and appreciation for multiple strategies, but they did not always use alternate strategies. Familiarity, understandability, efficiency, and form of the problem were all considerations for strategy choice. Practical and theoretical implications are discussed.
\end{abstract}




\section{Exploring the Development of Flexibility in Struggling Algebra Students}

For decades, researchers in the fields of mathematics education and cognitive psychology have been interested in the relationship between procedural and conceptual knowledge (e.g., Byrnes \& Wasik, 1991; Hiebert, 1986; Rittle-Johnson, Siegler, \& Alibali, 2001). Researchers have explored how these two types of knowledge are linked, including which develops first and whether one is necessary for the other. Yet, some suggest the dichotomy is not particularly useful for describing mathematical knowledge and should be abandoned (Wu, 1999). Recent arguments have emerged that critically examine the procedural/conceptual knowledge framework, with an emphasis on challenging how the two knowledge types have been defined and measured (Baroody, Feil, \& Johnson, 2007; Blinded, 2008; Star, 2005, 2007). In particular, Star (2005, 2007) argued that procedural knowledge is often conceived of and measured in ways that are consistent with rote memorization, whereas conceptual knowledge often encompasses both superficial and deep knowledge. Star argued that procedural knowledge can also be deep, as it must be in order to flexibly apply solution methods.

The ability to flexibly solve problems is considered an important outcome for school mathematics (National Advisory Panel, 2008) and is the focus of this paper. According to the National Council of Teachers of Mathematics (NCTM, 2006), students should have the ability to be strategic and efficient when solving simple equations. The National Research Council (NRC, 2001) argues for procedural fluency, which they define as "knowledge of procedures, knowledge of when and how to use them appropriately, and skill in performing them flexibly, accurately, and efficiently" (p. 121). Apparently, flexibility is a critical component of procedural fluency, but it is currently unclear how flexibility should be situated within the conceptual/procedural knowledge framework. Is flexibility part of procedural knowledge (Star, 2005) or part of 
conceptual knowledge (Baroody, Feil, \& Johnson, 2007), or does flexibility result from the integration of conceptual and procedural knowledge (Baroody \& Dowker, 2003)? We adopt the latter view in this paper, where we see flexibility as resulting from a merging of both procedural and conceptual knowledge.

Of course, flexibility is not a new construct. Its roots can be found in the seminal work of Krutetskii (1976) and Wertheimer (1959), and for years it has been considered an important element of adaptive expertise (Baroody \& Dowker, 2003; Hatano \& Inagaki, 1986). More recently, it has received significant attention from European researchers, who have been interested in children's flexibility with arithmetic (Blöte, Klein, \& Beishuizen, 2000; Blöte, Van der Burg, \& Klein, 2001; Klein, Beishuizen, \& Treffers, 1998; Torbeyns, Verschaffel, \& Ghesquiere, 2006; Verschaffel, Luwel, Torbeyns, \& Van Dooren, 2007). Perhaps because of these varying interests, definitions of flexibility have also varied. According to Verschaffel and colleagues (Verschaffel et al., 2007), some researchers equate flexibility with adaptability, while others make a distinction between the two constructs. Some researchers use the term to describe the ease with which a person can switch between various solution methods, whether or not the methods are optimal, while others suggest that a tendency to choose the optimal strategy in a given situation is a critical characteristic of flexibility. In the current study we take the latter perspective, suggesting that flexibility develops slowly from knowledge of multiple procedures to the adaptive use of them (Blöte, Van der Burg, \& Klein, 2001; Star \& Seifert, 2006).

It is important to note here that in this paper, strategy is used almost interchangeably with method or procedure (Siegler, 1996). However, trivial differences in solving an equation can technically result in a different procedure (e.g., adding a negative 5 as opposed to subtracting 5), whereas a strategy captures the essence of the procedure (e.g., using the quadratic formula). 


\section{Prior Research on Flexibility}

The literature on flexibility has begun to provide suggestions on both how to help students develop this capacity and also how researchers can assess it. Several recent studies found that students gained knowledge of multiple strategies for solving problems before they began to use them regularly (e.g., Blöte et al., 2001; Star \& Rittle-Johnson, 2008), which suggests it may be necessary to prompt students in order for them to fully demonstrate their knowledge. As an example, Blöte and colleagues showed that young students can learn multiple ways of solving addition and subtraction problems, but the tendency to use alternative methods lags behind their knowledge and preference for them (Blöte et al., 2001). In their study with 206 second graders, students involved in an instructional program that encouraged them to create and share solution methods showed higher levels of flexibility and conceptual knowledge than those involved in a program that emphasized skill and traditional solution methods.

Related to this work, Klein and colleagues (Klein et al., 1998) demonstrated positive effects of explicit flexibility instruction in their study of 275 second graders. These researchers compared the impact of arithmetic instruction that promoted flexibility from the beginning, to instruction that emphasized procedural skill prior to promoting flexibility. Both groups of students demonstrated flexibility at posttest; however, promoting flexibility from the beginning of instruction seemed to be more effective. Students who learned flexibility and skill simultaneously outperformed those who focused first on skill alone. Furthermore, their findings suggest that early exposure to multiple solution methods does not hinder procedural competence; the groups performed similarly on an arithmetic skills assessment.

Work by Star and Seifert (2006) also suggests that exposure to multiple ways of solving problems does not hinder accuracy. In their experimental investigation with middle school 
students, a treatment group $(\mathrm{n}=18)$ was asked to find two different ways to solve the equations presented to them, while a control group $(n=18)$ was asked to solve a series of equations in one way. While both groups were similarly accurate in their equation solving, the treatment group proved more flexible in their strategy use.

Using a related instructional intervention, research by Rittle-Johnson and Star (2007; see also Star \& Rittle-Johnson, 2009) demonstrated that students who are learning algebra can gain conceptual knowledge, procedural knowledge, and flexibility simultaneously. By comparing and contrasting two different worked solutions to the same problem, students in a treatment group (n =36) gained more on measures of flexibility and procedural knowledge compared to a control group $(n=34)$ that examined the same two methods but with isomorphic problems. Students in each group gained similarly with regard to conceptual knowledge. However, the study was conducted over four instructional days (within 45-minute classes), with only two of these involving the experimental intervention. Given this brief intervention, the significant differences are surprising, but it is not clear how flexibility with algebra might develop over time.

Finally, Star and Rittle-Johnson (2008) examined the development of flexibility by comparing direct instruction to a more discovery-oriented approach. In their study of 132 rising seventh graders learning to solve linear equations, they found that the different types of instruction had similar effects on accuracy of equation solving but differential effects on flexibility. Namely, prompting students to solve problems in more than one way was most effective for increasing students' use of multiple methods, whereas direct demonstration of efficient methods was most effective at increasing students' use of efficient methods. But although the treatments impacted flexibility differently in terms of $u s e$, they were similarly effective in increasing flexibility in terms of knowledge (i.e., knowing that multiple methods 
exist and that some are more efficient than others.) This distinction is important and lends support to the notion that flexibility develops on a continuum.

\section{The Current Study}

The current study builds on prior research on flexibility and addresses some of the limitations of that research. First of all, most prior studies have focused on elementary and middle school classrooms; studies examining flexibility in classroom settings at the high school level are lacking. Teachers in mathematics classrooms at this level are often faced with pressures to cover large amounts of material in a limited amount of time, grading systems that emphasize accuracy over efficiency, and a wide range of student abilities. Each of these features could possibly inhibit a focus on flexibility. Moreover, Star and colleagues (Rittle-Johnson \& Star, 2007; Star \& Rittle-Johnson, 2008; Star \& Seifert, 2006) have demonstrated that particular instructional techniques can be effective in promoting flexibility with certain types of linear equations, but they have done so within controlled environments using brief interventions. An important next step is to examine the feasibility of promoting flexibility in the context of an ongoing class, in which there is a limited amount of time to cover a set amount of material and students are held accountable for learning that material. The current study attempts to promote flexibility on a wide range of topics, throughout a three-week summer algebra course.

Additionally, few studies have examined how flexibility might develop for students who struggle with mathematics. Klein et al. (1998) reported that weaker second grade students were not confused by being introduced to multiple ways of solving addition and subtraction problems, but it is unclear whether or not this finding would hold for weak algebra students, who may have struggled for years and built up a number of misconceptions that could impede learning. In addition, within the field of special education there is considerable skepticism about the benefits 
of an approach focusing on multiple solution methods (Baxter, Woodward, Voorhies, \& Wong, 2002; Woodward \& Baxter, 1997); many feel that low achieving students would be overwhelmed by the cognitive load inherent in such an approach. The current study explores this issue directly by attempting to promote flexibility specifically among students who struggle with algebra.

Finally, most studies have relied exclusively on written assessments to understand flexibility. Although some of these assessments have included non-traditional items in order to make distinctions between knowledge and use of alternate methods (e.g., Star \& Rittle-Johnson, 2008), they have not examined why students make their particular strategy choices. The current study included interviews in order to better understand students' thinking about multiple strategies. For example, research suggests that students gain knowledge of alternate, efficient procedures prior to regular use of them (Blöte et al., 2001; Star \& Rittle-Johnson, 2008), and interviewing students may help researchers understand why this is the case.

The current study builds on prior work by using techniques that have shown to be effective in promoting and assessing flexibility (Rittle-Johnson \& Star, 2007; Star \& RittleJohnson, 2008). For example, having students contrast cases before discussing them may serve to deepen conceptual understanding by illuminating important features (Schwartz \& Bransford, 1998). In the current study, students compare and contrast pre-worked examples of solved equations. In order to distinguish knowledge of alternate methods from their use, students in the current study are sometimes prompted to solve problems in more than one way (Star \& Seifert, 2006). Building on prior work by including these features regularly within a classroom will provide both practical and theoretical contributions to the field. 
Two research questions guide the current study. First, in what ways do knowledge and use of multiple methods change during an algebra course focused on promoting flexibility? Prior research suggests students may gain knowledge of alternate strategies prior to using them (Blöte et al., 2001; Star \& Rittle-Johnson, 2008). More generally, in the larger literature on strategy choice, children frequently exhibit utilization deficiencies (Miller \& Seier, 1994), where knowledge of strategies appears to be present but the ability to use these strategies is lacking. We seek to confirm, elaborate on, and extend these findings, with a particular focus on the knowledge and skills of struggling students.

Second, in what ways does prior knowledge of algebra impact students' flexible use of solution methods? Of particular interest is whether prior knowledge of a single strategy makes it more or less likely that a student will adopt new, alternative strategies. Some prior work suggests that familiarity with one strategy increases the likelihood that learners will become flexible in the use of multiple strategies (Rittle-Johnson, Star, \& Durkin, 2009). It seems likely that the processing load for novices to learn multiple strategies simultaneously overwhelms their working-memory capacity (Sweller, van Merrienboer, \& Paas, 1998). In addition to this empirical evidence, teachers also express concern about the high cognitive load inherent in introducing low achieving students to multiple new strategies at once (Woodward \& Montague, 2002). Thus familiarity with one strategy appears to be an important and necessary resource in learning new strategies. On the other hand, it may be the case that prior knowledge and fluency with a single, standard approach for solving certain kinds of problems makes it less likely that students will adopt alternative strategies. If a student already knows one strategy for solving a problem, prefers to use this strategy, and can execute it efficiency and accurately, he/she may 
have little reason to learn and use other strategies. The present study seeks to explore this important issue in greater depth, again with a particular emphasis on low achieving students.

Given our interest in low achieving students, we were also interested in the effect of deficiencies in students' prior knowledge on subsequent learning of multiple strategies for solving algebra problems. There is a common perception that students who lack certain kinds of prerequisite skills (i.e., fluency with basic number facts and/or weakness with fraction computation) will later struggle in algebra courses (National Mathematics Advisory Panel, 2008). Thus, one might predict that students who are weak in prerequisite skills will be challenged to develop knowledge of even a single strategy for solving certain algebra problems ${ }^{1}$, much less knowledge of multiple strategies for the problem. On the other hand, weak prerequisite knowledge might serve as a powerful motivator for students to learn alternative strategies that reduce the reliance on particular skills. Given the prevalence of prerequisite-for-algebra knowledge deficiencies in US students (National Mathematics Advisory Panel, 2008), it is important to explore the impact of these deficiencies on students' flexibility in algebra.

Method

The context for this study was a small, three-week remedial/review summer algebra course, taught by the first author. The course was intentionally designed to promote students' flexibility. Below we describe the student participants, course curriculum, and assessments given to students during the course, followed by the procedures and analysis.

\section{Participants}

Two boys and four girls, all of whom were enrolled in the same high school, participated in the study. Xavier, Ricardo, and Nicole were all new to the school and were entering the ninth grade. Because these students were new to the school, they were required to take a placement 
exam in algebra that suggested the need for a remedial course in algebra. The other three students were not new to the school, which meant prior teachers recommended them for the course. Annemarie and Naomi were entering tenth grade, and Yvonne was entering eleventh grade. All six students had taken a first course in algebra prior to the summer course. Four of the students took this first course during the school year that was just completed, but Naomi and Yvonne had taken it one year earlier and had just finished a year of geometry.

\section{Course Curriculum}

The three-week course included 14 instructional days plus one day devoted to the final exam. The class met Monday through Friday each week for two hours in the afternoon. The first week focused primarily on Unit I, which included solving linear equations, graphing linear equations and solving systems of equations. The second week focused primarily on Unit II, which included simplifying exponential expressions and simplifying radical expressions. Factoring, which was part of Unit III, was begun at the end of the second week. The final week continued with factoring and then focused on solving and graphing quadratic equations.

A typical lesson involved comparing and contrasting side-by-side worked examples of problems relevant to the day's topic. The characteristics of the worked examples varied in such a way that one method might be preferred in some cases, but a different method might be preferred in other cases. However, at no time in the course were students pressed to use a particular method. Instead, they discussed which methods they preferred, why they preferred them, and under what circumstances. For example, students might be presented with problems of the type $a(x+b)=c$ that are solved by using the distributive property as a first step and then solved by "clearing" $a$ as a first step. Variations might include $a$ being a fraction, $c$ being divisible by $a$,

and $a$ and $c$ being relatively prime. To illustrate, it might be preferable to solve $\frac{2}{3}(x+5)=8$ and 
$3(x+1)=15$ by "clearing" $a$ first by multiplying or dividing both sides of the equation by a constant. Alternatively, it might be preferable to distribute first for the isomorphic problem $4(x+$ $1)=19$. For these choices, discussions might focus on the number of steps it takes to solve the problem, the ease or difficulty of computation, or the divisibility of certain factors. In other words, worked examples were designed to draw students' attentions to the structure of the equations rather than the superficial characteristics (e.g., that an equation included parentheses), and discussions focused on ways that the structure might indicate which methods were possible or optimal. However, students were not expected to always agree on which methods they preferred.

Table 1 shows the side-by-side format that was used to present worked examples to the students, who examined them in pairs. Before discussing the methods, they were asked to first determine what strategy was used to solve the problems. In these cases, all problems within a single column were solved using the same strategy. As the course progressed, students were sometimes asked if they knew of more than one way to solve a problem rather than being shown two ways and comparing them. This transition was meant to scaffold students, allowing them to consider and create multiple solution methods on their own (see Star \& Seifert, 2006). Table 2 shows sample algebra problem types and some of the strategies that received attention in the course.

[insert Table 1 here]

[insert Table 2 here]

It is important to note that in order to promote flexibility, various strategies were introduced and discussed with an emphasis on meaning. For example, after a student demonstrated how to use the distributive property to solve $5\left(j+{ }^{-} 9\right)=30$, the students were asked 
questions such as "I see how you got 5j, but how did you get negative 45? Why did you multiply the 5 by negative 9?" When they responded with a rule only, they were probed further with questions such as "If we multiply a term by 5, what does that mean? In this case, what would be added five times?" until the expansion $5\left(j+{ }^{-} 9\right)=\left(j+{ }^{-} 9\right)+\left(j+{ }^{-} 9\right)+\left(j+{ }^{-} 9\right)+\left(j+{ }^{-} 9\right)+\left(j+{ }^{-} 9\right)$ was written on the board. As another example, problems in the form $x^{\mathrm{a}} \cdot x^{\mathrm{b}}$ were expanded (e.g., $\left.m^{2} \cdot m^{4}=m \cdot m \cdot m \cdot m \cdot m \cdot m\right)$ in order to help students understand why the exponents were added. This emphasis on meaning was an integral part of the instruction throughout the course.

\section{Measures}

Algebra exam. The final exam for the course served as both a pretest and a posttest. It included 55 items and was adapted from an exam designed by an experienced algebra teacher in the school. Topics on the exam included linear and quadratic equations, systems of equations, graphing, and pre-requisite skills for quadratics such as factoring and simplifying with exponents and roots. In order to assess students' flexible use of strategies, some of the items were designed such that the most commonly taught approach might not be the easiest or most efficient.

To help validate that these items, the test was given to eight experts, including two mathematics teachers, two mathematics educators, two mathematicians, and two engineers. The experts took the test and were interviewed about the particular problems designed to test flexibility. They were asked why they chose the method they did, whether they knew of another method, and which method they preferred and why. Results from the experts suggest there is a strong tendency to prefer methods that are "easy." When asked to define "easy" they consistently used phrases such as "faster", "fewer steps", and "less complicated." The current paper uses efficient as a way to summarize these various explanations, but this issue is explored more carefully in the section addressing theoretical implications of the study. There was surprising 
consistency in the methods that experts preferred for the items designed to test flexibility. In general, experts departed from general algorithms where appropriate and they cited structural characteristics of the problems to justify their choices (see Blinded, 2009). Because the experts demonstrated flexibility on the expected problems, no changes were made to the final exam. Intermediate assessments. The course was divided into three units, and students were assessed accordingly. The first unit focused on solving and graphing linear equations. The second unit focused on simplifying with exponents and radicals. The final unit was focused on solving and graphing quadratic equations. Each unit included daily homework, one quiz, and one test. Unlike the final exam, the intermediate assessments sometimes prompted students to solve problems in more than one way (Star \& Rittle-Johnson, 2008; Star \& Seifert, 2006). Students were prompted to solve problems in more than one way in order to determine whether or not they had knowledge of solution methods that were different than the ones they chose to use on their own, without being prompted. On these problems, students were asked to indicate which method they thought was better by placing a star by that method. For each problem where students were asked to generate multiple methods, an isomorphic problem (where students were only asked to generate a single solution method) was included as well, to indicate students' preferred method for that problem. Typically, the isomorphic problems appeared earlier in the assessment than the prompted problems, so that students were not likely to be influenced by the prompt.

Interviews. The pre-interview and post-interview were identical, and they prompted students to solve problems in more than one way or to evaluate two methods of solving or graphing. To illustrate, students were shown the equation $3 x+2 y=9$ and asked the following sequence of questions: a) What is one method I could use to graph the equation? Can you show 
me how to do it that way?; b) Can you think of another way to graph the equation? Can you show that method?; and c) Is one of the methods better than the other? Which one? Why do you think it is a better method? Students were also asked about strategies for solving linear equations, quadratic equations and systems of equations. Finally, students were asked about their views on learning more than one way of solving problems. The intermediate interview asked students to elaborate on their solution methods from the first test in the course, and it also asked about their views on learning to solve problems in more than one way. Each of the three interviews lasted approximately ten minutes.

\section{Procedure}

Students were interviewed before the course began, immediately following the first test, and after the final exam. The interviews were audiotaped and later transcribed. The pretest was administered on the first day of class, and the posttest was administered after the last day of class. A quiz and a test were administered for each of three units, and homework was assigned nearly every evening. All assessments were photocopied for subsequent analysis before being returned to the students. All students and their parents consented to participation in the study. At the end of the three weeks, students were provided with a small gift card to a bookstore. Analysis

The analysis was guided by prior research. In particular, the data were examined for relationships between knowledge and use of alternate methods and the role of efficiency when choosing a solution method. Exploration of the data began with the coding of students' strategies within each problem type, in order to determine which strategies students used for each problem on the pre-test, post-test, unit tests, and homework (including problems that were designed to assess flexibility). Because strategies differ with problem types (i.e., strategies for graphing 
linear equations cannot be used to solve quadratic equations), the major problem types were analyzed separately. Graduate students and the second author drew from prior research to develop an a priori strategy typology for each problem type, which was used to identify when students used standard and alternative solution methods. This phase of the analysis yielded a profile of strategy usage for each student on each problem type, including students' strategy repertoire and distribution (Lemaire \& Siegler, 1995). Subsequently, students' interviews were analyzed in an attempt to understand, confirm, and/or challenge students' strategy profiles as found in the written data. Emergent themes from students' interviews were identified by graduate students and the second author, with subsequent discussion and confirmatory analysis from the first author (who taught the course). Finally, cases were constructed that elaborated on students' knowledge of and flexible use of multiple solution strategies, including attitudes toward flexibility and rationales for strategy choices.

\section{Results}

The results section is organized around our two research questions relating to students' flexibility: a) changes in students' knowledge and use of multiple solution methods during the course, and b) the impact of prior knowledge of algebra on students' flexible use of solution methods. But first, we begin by briefly reporting on students' performance and strategy use at the beginning of the course.

\section{Performance and Strategy Use at Pretest}

Recall that all six students had taken a year-long algebra course prior to the summer course and were either recommended by teachers to take the summer course or were required to take it based on results of a placement test. As expected, no student demonstrated mastery of the material at pretest. Although all students showed familiarity with simplifying expressions and 
solving linear equations, they often made errors. Many of the problems assessing knowledge of advanced Algebra I topics, such as solving quadratic equations, were not attempted. For example, none of the six students attempted to solve problems involving systems of equations, indicating weak or no knowledge of how to approach this problem type. In addition, Xavier, Ricardo, and Nicole left all quadratic problems blank, indicating weak or no knowledge of this topic at the beginning of the course. Yvonne had moderate knowledge of three solution methods for approaching quadratics. Namely, she demonstrated knowledge of solving by factoring, by taking the square root of both sides of the equation, and by using the quadratic formula (see Table 1). Annemarie and Naomi had limited knowledge of solving quadratics; each successfully solved 1 out of 6 quadratic equations on the pretest.

When probed about their knowledge of multiple strategies during the pre-interview, students generally indicated knowledge of only a single strategy for solving many types of algebra problems. For example, all six students demonstrated knowledge of one strategy for solving linear equations such as $5(3 x+1)=8$. As Yvonne stated, "You have to distribute first." For graphing linear equations, two students did not remember any strategy. The other students only knew of one strategy, which is the one described by Ricardo:

[Do you remember some ways to graph lines?] Yes. [How do you graph an equation like this? $(3 x+2 y=9)]$ You plug in the numbers for $x$ and $y$. [And then what?] And you plot them. [Can you think of any other way to graph this?] No. (Ricardo, pre-interview)

In only a few cases did students indicate knowledge of more than one strategy for solving algebra problems. In particular, pre-interviews revealed that Annemarie, Yvonne, and Naomi knew more than one strategy for solving quadratic equations. For example, in Naomi's preinterview about the quadratic formula, she admitted to "vaguely" remembering three methods to 
solve quadratics, but preferred factoring "because the quadratic formula takes a long time to plug everything in and then solve it." Naomi's statement suggests that she considered efficiency when deciding which method to use for solving quadratics equations; she was the only student who alluded to efficiency at pretest. Annemarie's pre-interview suggests she also knew of the quadratic formula prior to the course but preferred factoring "because if you don't understand math that well, it's easier for me than the quadratic formula." For her, understandability was a key. Yvonne, on the other hand, suggested that the quadratic formula was often the best method for solving quadratic equations, seemingly because of its straightforwardness - that you just need to "plug [the values] into the formula."

As a whole, and despite the fact that all six students had taken a year-long algebra course prior to this study, students had quite weak knowledge of strategies for solving a wide range of algebra problems. Pretest scores support the notion that the students struggled with algebra. Both pretest and posttest scores for each student are provided in Table 3.

[insert Table 3 here]

\section{Knowledge and Use of Multiple Methods}

Our results suggest two findings with respect to students' knowledge and use of multiple methods. First, knowledge tended to precede use for the most familiar problem types. Second, two primary reasons were given when multiple strategies were regularly used. Namely, students considered the symbolic form of problems and (to a lesser degree) the relative efficiency of applicable strategies when deciding which methods to use for a given problem.

Knowledge versus use. First, across the most familiar problem types there was evidence that students' knowledge of alternate methods preceded their use of them. The first test in the course (Test I) provides an illustration of how knowledge preceded use for linear equation 
solving. Recall that intermediate assessments contained questions where students were asked to solve a problem in more than one way, as well as isomorphic problems that only asked for a single method. For example, on Test I students were asked to solve $7(n+2)=49$ in only one way, while later in the test they were asked to solve $-5(7 x-16)=45$ in more than one way. The results from Test I indicated that all six students solved $7(n+2)=49$ by distributing the 7 as a first step (a standard approach that was discussed during the course). Yet, when students were prompted to solve $-5(7 x-16)=45$ in two different ways, all six showed knowledge of both the standard approach and the alternative strategy (multiplying or dividing by a constant as a first step). Some students indicated a preference for this alternative method, despite the fact that they did not use it without being prompted.

Students were somewhat more likely to use alternatives to the standard approach with problems that posed difficulty, such as those containing fractions. For example, three students solved $\frac{1}{2}(x-15)=-4$ using the standard approach (distributing the $\frac{1}{2}$ as a first step), while the other three chose to use an alternative strategy that was introduced in the course - multiplying both sides by the reciprocal of $\frac{1}{2}$, or 2 . As Xavier noted for this problem, "I think since there is a fraction, it would be easier just to use the reciprocal, in this case, instead of just working out the whole distributive property" (Xavier, intermediate interview). These strategy choices are further discussed in later sections.

As with solving linear equations, students also seemed to gain knowledge of alternate strategies for simplifying radical expressions before they tended to use them. For example, when simplifying radical expressions in the form $\sqrt{\frac{a}{b}}$, a general approach is to take the square root of 
the numerator and denominator separately, and then to simplify the result. However, in the case that $\frac{a}{b}$ is equal to a perfect square, it can be more efficient to simplify the radicand before taking the square root (see Table 1). On Test 2, all six students demonstrated knowledge of this alternate strategy for simplifying radical expressions, but only three used it without being prompted (to simplify $\sqrt{\frac{50}{2}}$ ). Interestingly, two of the three who used the general approach on Test 2 made errors and subsequently changed their strategy. On the posttest, these two students were the only ones to use the alternate strategy without being prompted (to solve $\sqrt{\frac{75}{3}}$ ). Once again, it seemed students were more likely to switch strategies for difficult problems. The students had not only switched strategies, but they had also improved in accuracy. On the other hand, one student who used the general approach on the posttest made an error.

Recall that all students showed familiarity with solving equations and simplifying expressions on the pretest. For these topics, students were generally more likely to use the familiar, general approach unless this approach proved difficult. For more advanced topics where students demonstrated weak knowledge at posttest, students more easily switched between strategies. Reasons for students' strategy choices are described in the following section.

Reasons for strategy choices. A second result relating to knowledge and use of multiple strategies concerns the criteria used by students when selecting strategies for solving problems. During interviews, the efficiency of known strategies and the symbolic form of the particular problems-to-be-solved were repeatedly identified as factors driving the selection of strategies for the least familiar problem types (based on the pretest). 
Students most frequently described the form of the equation when providing a rationale for their selection of strategies, as was the case when solving systems of equations. For example, when one of the equations was already solved for $y$ (e.g., the system of equations for problem 19 included $x+y=8$ and $y=3 x$ ), students generally chose to substitute its equivalent expression into the remaining equation:

[Let's look at systems of equations. What was your method for number 19?] Oh, substitution because one of the systems was $y=3 x$ and it's right there, what $y$ equals, so you just substitute. (Xavier, intermediate interview)

Likewise, Yvonne used the substitution method "because it seemed right, because it was already $y=3 x "$ (Yvonne, intermediate interview). Ricardo and Naomi gave similar reasons for using substitution for this same problem.

For the next problem (the system of equations $x+y=15$ and $2 x-y=12$ ) most of the students switched methods, choosing to add or subtract the equations so that one of the variables canceled (i.e., the "elimination method"). For example, Naomi used the elimination method "because there were already two opposites, positive $y$ and negative $y "$ (Naomi, intermediate interview).

Form was also mentioned when explaining choices for graphing linear equations. For example, although Ricardo chose to graph a line by plotting points when "the problem is really easy", such as for $x+y=10$, he switched methods when the form of the equation was more conducive to a different method:

[What method did you use to graph this? $(x+y=10)] \mathrm{Um}$, I just plugged in numbers. [You did?] Yeah. [What about for this one? $\left(y=\frac{1}{2} x+3\right)$ ] Since it already had the slope and the $y$ - 
intercept, I just found the $y$-intercept and went up from there, using the slope. (Ricardo, intermediate interview)

Similarly, Xavier's rationale for graphing lines using the slope-intercept method was that "you know where to start, and from there you just to use the rise over run; it is ready to go" (Xavier, intermediate interview). And Nicole, who admittedly struggled with all graphing methods, successfully used the slope-intercept method for the same problem "because it was basically in that form" (Nicole, intermediate interview).

In some cases, efficiency was cited when explaining choices for graphing linear equations. For example, Naomi and Yvonne both successfully used the slope-intercept method rather than plotting points on Test I. Although Naomi's intermediate interview revealed she had knowledge of both methods, she said she used the slope-intercept method "because it's faster." Likewise, Yvonne said her reason for not choosing to plot points was that "there is more involved, and it takes longer."

For quadratic equations, students' decisions about strategies were also made using a mix of efficiency and form at posttest. In her post-interview, Annemarie highlighted efficiency as a rationale for her choice of methods. For example, she said that she preferred the square root method (see Table 1) in problems with no $x$ term "because it's faster." Naomi mentioned fewer steps as a reason to use factoring instead of the quadratic formula. Likewise, Xavier preferred not to use the quadratic formula because, for the other two methods, "there are less steps and it's just, there's a clear way to get the answer" (Xavier, post-interview). He also mentioned the form of the equations, stating that one equation had a "perfect square" and another one was "pretty easy to factor, it's already in a factorable form." Presumably, Xavier was referring to the fact that the equation was already set equal to zero. Nicole, who often preferred the quadratic formula 
because she admittedly struggled with factoring, also attended to form. In her post-interview, her reason for preferring factoring for one problem was "because there's no coefficient" in front of the squared term.

\section{Impact of Prior Knowledge on Flexibility}

Our results suggest three findings with respect to students' prior knowledge of algebra. First, fluency with a single strategy seemed to inhibit flexibility. Second, when knowledge of algebra or its pre-requisites was particularly weak, students were more likely to use alternative strategies, but with a focus on accuracy and understandability rather than efficiency or form. Finally, students' weak prior knowledge seemed to be related to positive views of learning multiple strategies for solving problems.

Prior knowledge of single strategies. As noted above, the prior literature offers suggestions for ways that prior knowledge of algebra may help or hinder the development of flexibility. Our results suggest that prior knowledge of single strategies largely exerted a negative influence on the development of students' flexibility, especially when students began the course with considerable fluency with those strategies. The most prominent example of this trend concerned linear equations of the type $a(x+b)=c$ where $a$ was a whole number. The course attempted to help students develop flexibility for solving problems of this type, presenting them with an alternate to "distribute first" when $c$ was divisible by $a$ (i.e., use the "divide first" method; see Table 1). Several students began the study having already developed fluency with the "distribute first" method for these equations. Yet it appeared that students' prior instruction had focused so exclusively on this strategy that it may have hindered their ability to use other strategies for problems of this type.

In particular, written assessments reveal that, at pretest, every student in the class except 
Xavier solved problems of the type $a(x+b)=c$ where $a$ was a whole number by using the "distribute first" method. (At pretest Xavier solved problems of this type incorrectly, and it is unclear what method he used since he did not show any work.) During the course, all students gained knowledge of the alternative "divide first" method for this problem type and could demonstrate how to solve problems using this method when prompted to do so. However, when given the opportunity to solve problems using the method of their choice on homework assignments and assessments, students in the course were quite consistent in continuing to use their original preferred "distribute first" method.

Students' interview responses provide insight as to why knowledge of the "divide first" method did not lead consistently to its use. To illustrate, Yvonne indicated in her immediate interview that she had now learned a second way to solve problems of this type, the "divide first" method. Yet she explained that on a recent exam problem, she still used the familiar "distribute first" method because "it was like instinct":

[Let's look at how you solved some of the problems. This one here? $(7(n+2)=49)]$ I distributed the 7 to the $n+2$. [Distributed. Okay. Why did you choose that as your first step?] Um, just because it was what came to mind first and it just seemed easiest. [Do you know another way to do it?] You could divide the 49 by 7 and just get $n+2=7$, and then you would get $n=5$. [And which way do you like better?] The first way. Just the way I did it. I mean, I guess it doesn't matter, but it was like instinct, sort of. (Yvonne, intermediate interview)

Yvonne frequently described her use of the "distribute first" method as being "like instinct" and further indicated that she had developed fluency with this method from frequent prior instructional exposure. This fluency may have deterred her from examining test problems 
and considering whether alternative methods might be easier in some way. Annemarie's and Ricardo's interview responses show a similar pattern. During the intermediate interview, Annemarie explained that while she knew how to use the alternative "divide first" method, she had still solved a problem of this type on a recent test by using "distribute first" because "It's just the way I was taught." Similarly, Ricardo demonstrated during the intermediate interview that he could also apply the new "divide first" method he had learned, but he indicated that he still preferred to use "distribute first" because he was "used to it."

Weak knowledge of algebra. Unlike the other students, Naomi demonstrated prior knowledge of an alternate strategy for solving linear equations, at least for those involving fractions. In particular, she stated that one of her teachers taught her to "clear the denominator" when solving equations with fractions. For example, at pretest she solved $\frac{2}{3} w+3=10$ by first multiplying all terms by 3 , as illustrated in Figure 1:

[insert Figure 1 here]

For the same problem at posttest (see Figure 2), Naomi used the more traditional first step of subtracting 3 from both sides but then again cleared the denominator by multiplying by 3 :

[insert Figure 2 here]

In both cases, her alternate strategy was arguably less efficient than the typical method of subtracting 3 and multiplying by the reciprocal. In other cases it was more efficient, as a matter of coincidence. Ostensibly, Naomi's focus on eliminating fractions was a way to reduce the likelihood of making errors.

In fact, the presence of fractions in a problem was often a key factor in leading students to change strategies, as a result of students' discomfort with working with fractions. In particular, fractions were often cited as a reason to not to distribute on linear equations of the form $a(x+b)$ 
$=c$. Ricardo, who expressed a preference for distributing for most problems of this type, felt differently when $a=\frac{6}{5}$. In this case, he preferred to multiply by the reciprocal of $\frac{6}{5}$, "because this one was totally confusing. To distribute was really confusing. It was getting confusing multiplying $\frac{6}{5}$ by this. It was easier just to get rid of it" (Ricardo, intermediate interview). To solve $\frac{1}{2}(x-15)=-4$, Nicole chose not to distribute the $\frac{1}{2}$ "because I feel as though I'm not, sometimes not good with fractions" (Nicole, intermediate interview). When Naomi was asked about her solution to the same problem, she stated that she preferred to multiply both sides by 2 instead of distributing the $\frac{1}{2}$, "so that you don't have to work with fractions."

In general, weak knowledge of algebra or its pre-requisites prompted the use of alternate strategies for solving problems, but this use was motivated by a concern with accuracy or understandability. As another example, students in the class learned two ways to simplify expressions when multiplying with the same base (e.g., $a^{3} \times a^{4}=a^{7}$; see Table 1). One method was to expand all the terms and then find a single term that was equivalent to the expansion. The second method was to add exponents and keep the base the same. In this case, adding exponents is the general strategy and arguably the more efficient one, but some students were not comfortable with that method and chose to expand the terms in order to simplify the expression, as demonstrated by Xavier in Figure 3:

[insert Figure 3 here]

When simplifying more complicated expressions, some students still used the expansion method. For example, in Figure 4 Annemarie seemed to use the method to verify that exponents could be 
multiplied when raising a power to a power. Although Annemarie's method was inefficient, it was understandable to her and allowed her to solve the problem correctly:

[insert Figure 4 here]

Although weak knowledge often led students to use inefficient methods, it sometimes led to efficient ones. For example, Annemarie's reason for using the slope-intercept method for graphing $x+y=15$ was that "the T-chart is really confusing for me for some reason." For $y=\frac{1}{2} x-3$, she again used the slope-intercept method, stating that it was because "the chart is too confusing with a fraction." When choosing not to distribute for certain problems she said, "Distributing fractions kind of scare me. Distributing them, I'm not good with fractions so I was afraid that if I distributed it, I would distribute the fractions wrong" (Annemarie, intermediate interview). Again, a concern with accuracy was the driving force for strategy choices in cases where prior knowledge was weak.

Students' views of learning and using multiple strategies. Post-interviews support the notion that students in the course who struggled with basic algebra and rational number skills were often more concerned with accuracy rather than efficiency. For example, when asked about disadvantages of learning multiple methods in the post-interviews, the same possible disadvantage was offered by all six students. As Xavier stated, "Sometimes you could get the two methods mixed up." However, it was somewhat surprising that five of the six students insisted this was simply a possibility for someone else. As illustrated by Naomi, "People could do that, but I don't tend to get methods confused. Either I get it completely right, or I forget the method all together." Only Annemarie admitted that she might personally "mix up different methods." At the same time, however, she also expressed that the first method she learned did not always make sense to her. In those cases, learning a new method was particularly helpful: 
"[What are some advantages to learning more than one way?] There were, like with the radicals, how you showed me two methods. My teacher taught me, my old teacher taught me one method, and it was a method that I really didn't understand. And when you showed me the other method I was like, wow, this is easier. And I could understand it more and I always hated radicals, but now I am starting to get them." (Annemarie, post-interview)

Annemarie expressed a similar perspective during the intermediate interview, when solving systems of equations. Although Annemarie showed knowledge of two methods, she was more comfortable with substitution:

"[So why did you use the substitution method here?] Because my teacher taught me the elimination method and it always kind of struck me really confusing and I actually was never good at this, I always used to get them wrong. And when he told me about substitution, it became easier to me because it was easier for me to see how you substituted it." (Annemarie, intermediate interview)

The focus on accuracy may partially explain why knowledge of efficient methods sometimes preceded the regular use of them; if the method first learned by the students was not difficult to apply for a particular problem, they tended to continue using it. But despite not always choosing to use alternate methods, students in this study still generally viewed their knowledge of multiple strategies as advantageous. As suggested by Nicole, an alternate method can serve as a "backup" method if needed: "[And what are some advantages to learning more than one way to solve math problems?] Well it's an advantage because in case you get stuck doing one way, you can always have a backup" (Nicole, post-interview). Ricardo had a similar response, suggesting that "If you get stuck on one way then you can do it the other way." Xavier and Yvonne made similar suggestions and added that being able to check your work was another 
advantage of having an alternate way to solve a problem. Only Naomi alluded to efficiency as an advantage to knowing more than one way to solve math problems. She said, "Well knowing more than one way makes it easier to solve an equation just because one way might make solving it a lot harder, so if you know a different way, you could do it faster."

\section{Discussion}

Being able to flexibly solve problems is one of the hallmarks of procedural fluency (National Research Council, 2001), and recent attention to flexibility in the research literature has revealed some interesting findings about its development. The current study confirms and adds to those findings in several ways. The purpose of the study was to explore the development of flexibility with algebra within a classroom setting and with students who struggle with algebra.

First, our findings confirm that although students may have acquired knowledge of alternate methods, possessing a knowledge of alternate methods does not necessarily imply that students will tend to use them (Blöte et al., 2001; Star \& Rittle-Johnson, 2008), particularly if they have gained fluency with one particular method. For example, in the current study, students commonly explained their use of the distributive property as something they employed because they were "used to" it, even when they acknowledged it was not the most efficient method. It seems that being overly practiced with one particular method for a problem type actually inhibited flexibility with that type. These results are consistent with Klein et al. (1998), who found that second graders who focused on skill before flexibility were less flexible that ones who focused on both at the same time. The finding may be explained by the Einstellung effect, which suggests that people tend to approach problems in familiar ways, even when better methods are available (Luchins, 1942). However, research by Rittle-Johnson et al. (2009) suggests that some 
familiarity with a single approach is necessary before students can benefit from the comparison of multiple approaches, possibly because it eases the cognitive load necessary for comparison. A question that remains is, under what circumstances is the exposure to a single method considered too much? When does it impede flexibility as oppose to facilitate it?

Second, when students encountered problems that were especially difficult or confusing for them, they were more likely to use a new method for that problem. This finding is somewhat counter-intuitive, since one might expect struggling students to prefer to rely exclusively on one broadly applicable strategy. Yet, students in this study seemed grateful to have a "backup" method. This finding was especially true for problems involving fractions; students consistently named being "not good with fractions" as a reason to avoid a particular method, both for solving and graphing equations. In general, weak knowledge prompted the use of alternate strategies, but a concern with accuracy or understandability was often the driving force for deciding on which strategy to use.

Finally, when two methods were equally familiar and comfortable, students were likely to cite efficiency and/or equation form as reasons for choosing particular methods. For example, students demonstrated little or no knowledge of solving systems of equations at pretest, but after learning two methods for solving systems, most students used and switched easily between the two methods during the course. During the intermediate interview, students consistently suggested that they chose a particular method because of the way the problem was posed to them.

In brief, familiarity, understandability, efficiency and form were typical reasons given for strategy choices. Results of the current study hypothesize the following developmental progression with regard to flexibility: a) using what is familiar and reliable; b) attempting 
alternate methods (but not necessarily efficient ones) when challenged with a difficult or confusing problem; and c) using methods that are efficient and/or take advantage of the problem's structure or form. More research is needed to test this hypothesis.

Recall that the posttest was also administered to a group of experts (Blinded, 2009). These experts demonstrated flexibility in terms of both knowledge and use of strategies, and they were in surprising agreement about which methods were optimal. When prompted to explain why they chose their methods and whether they knew of other methods, they consistently pointed to structural characteristics of the problem (e.g., a certain number is divisible by another) and preferred methods that were more efficient, made use of important mathematical ideas, and reduced the chance for error. In other words, the experts were primarily focused on form and efficiency. It seems that when students were not struggling with accuracy, they more closely resembled experts who took the same test.

\section{Implications for Theories of Flexibility}

The present study also offers theoretical insight into current conceptualizations of flexibility. In particular, and as noted above, there is variation in the literature in terms of how researchers define flexibility. However, there is agreement that flexibility involves the use of strategies that are considered the most appropriate for a given problem. But what does appropriate mean?

On the one hand, one could consider the most appropriate strategy to be the most efficient one. Certainly it is seems intuitive that efficiency should play a role in the determination of which strategy is best for a given problem. But a number of issues emerge when appropriateness is closely linked with strategy efficiency. First, efficiency itself is difficult to define. Is the most efficient strategy the one that can be executed the fastest? executed in the fewest steps (and what 
counts as a 'step')? executed with greatest ease? As one of the experts noted, "It's not about extra steps. I don't mind putting in extra steps if extra steps makes it easier" (Blinded, 2009, p. 17). Second, in some situations, the most appropriate strategy may not be the most efficient one. For students who struggle with mathematics, the most appropriate strategy might be the one that is the most understandable in a given situation. And finally, equating flexibility with efficient problem solving can have unintended instructional consequences. As Verschaffel and colleagues suggest, if efficiency is the primary or only criterion for strategy selection, instruction can degenerate into a series of "'(quasi-)algorithmic' rule[s] for linking problem types to solution strategies and with systematic training in the use of [those] rules" (Verschaffel et al., 2007, p. 33). Hence, a focus on efficiency may not ultimately offer sufficient clarity in determining strategy appropriateness.

However, recognizing that appropriateness is more than efficiency leads to another potential challenge - conceptualizing appropriateness in a way that is not overly individualized (and thus difficult to generalize). Verschaffel and colleagues (Verschaffel et al., 2007) suggest that a strategy is most appropriate "on a given mathematical task, for a given individual, in a given context or situation" (p. 32). In some respects, this depiction of appropriateness is difficult to argue with. Certainly context, task, and person must play a role in determining which strategy is most appropriate for a given problem. On the other hand, this definition makes it difficult to formulate hypotheses about the appropriateness of strategies that generalize and thus can span multiple contexts, individuals, and tasks. Yet doing so seems critical for instruction focusing on flexibility. Furthermore, does appropriateness in such a case become reduced to an individual's strategy preference? If a solver can articulate a reason that his/her strategy choice is most 
appropriate on a problem (i.e., it is her preferred strategy or one that she understands the best), can we ever argue that her choice is not the most appropriate choice?

The current study offers a somewhat modified vision of what "appropriate" means that recognizes the importance of person, task, and contextual variables but also with an eye toward the need to formulate general instructional recommendations for students as they develop flexibility. In particular, students' strategy choices in the present study appear to be guided by an interaction between problem type, affordances/constraints of particular strategies, and prior knowledge. Problem type refers to the general structure of a problem, such as whether an equation is written in the form $a(x+b)=c$. Affordances and constraints of strategies indicate whether conditions in specific problems within a problem type impact the ease or efficiency with which strategies can be executed by an individual, such as whether $c$ is evenly divisible by $a$ in the problem type above. And prior knowledge refers to the knowledge that a student brings to the problem which impacts the affordances and constraints of known strategies. For example, if a student is quite fluent with fractions, then a particular strategy for the problem type $a(x+b)=c$ (i.e. dividing both sides of the equation by $a$ ) may be quite appropriate, even if $c$ does not evenly divide $a$. But for a student who struggles with fraction computation, this strategy seems appropriate only when $a$ is a factor of $c$. Similarly, when solving quadratic equations written in standard form, certain combinations of constants may suggest that factoring is the easiest strategy to perform, but individuals' prior knowledge related to factoring would certain impact the relative appropriateness of this strategy. Understanding these subtle interactions may be important considerations for instruction and can be useful to include in classroom discussions meant to promote flexibility.

Implications for the Teaching and Learning of Algebra 
The findings of this study have several implications for algebra classrooms. First, teachers need not avoid a focus on multiple strategies with struggling students, out of concern that students' lack of prerequisite skills will make flexibility an unattainable goal. Rather, our results suggest that low achieving students may be particularly appreciative and excited about a focus on multiple strategies. Knowing more than one way to solve a problem may enable them to choose a method that maximizes the accuracy of their solution and/or that is most clear or understandable to them.

However, when focusing instruction on multiple methods, teachers need to carefully choose the target examples and strategies in order for similarities, differences, and affordances of various approaches to be clearer. Specifically, problems should not differ on too many features and presentation should be side by side to promote comparison. Related to this, it is not enough for students to be exposed to multiple methods; discussion of similarities and differences in methods is critical (Schwartz \& Bransford, 1998). Students may need a chance to compare methods and learn the conditions, goals, and problem types that make certain strategies useful. Teachers should also use visual and gestural cues to help students see what is similar and what is different in problems or strategies (Richland, Zur, \& Holyoak, 2007).

Finally, if flexibility is to be an important goal of school mathematics (National Mathematics Advisory Panel, 2008), its assessment needs to be carefully considered. Given the fact that knowledge of multiple strategies precedes use, teachers need to devise assessments that not only capture use but also knowledge. For example, asking students to solve certain problems in more than one way can illuminate knowledge of multiple strategies even when they are not regularly being used. Doing so may also have the advantage of encouraging their use.

\section{Limitations and Future Studies}


Although the current study extends the work of Star and colleagues by promoting flexibility within a real class (i.e., one that involved regular homework, assessments, and grades), in many ways the class was not typical. First, the small class size created an environment more conducive to discussion, which served as an important vehicle for promoting flexibility. It could be more challenging to conduct similar discussions in larger classes. Similarly, the small number of students in a select setting also limits the generalizability of the findings; replication with larger groups and more diverse settings is needed. Furthermore, the class was held in the summer, lasting only three weeks. Although a small-scale examination of the role flexibility might play in an ongoing classroom was an important next step, future studies should be conducted in semester- or year-long algebra classrooms in order to assess long term effects of promoting flexibility. Researchers and educators need to understand how long it takes to move students from knowledge to use of alternate strategies, as well as what other benefits a long term focus might have for algebraic knowledge (e.g., deepened conceptual understanding). Research in this direction is currently underway.

Larger classes would also be useful in determining the impact of promoting flexibility on performance. Although performance was certainly relevant for the students in the current study (i.e., they were given grades for the course), it was not included as a specific outcome of interest. With regard to students who struggle, the current study suggests they appreciate and benefit from learning multiple strategies for solving equations but it would be interesting to compare these students to those who excel at algebra.

Future studies should also examine the impact of flexibility on attitudes and beliefs about mathematics. A focus on flexibility shifts the emphasis from correct answers to appropriate processes, which could possibly alter students' understandings of what it means to do 
mathematics, as well as their habits of mind about mathematics (Cuoco, Goldenberg, \& Mark, 1996). Although the current study did not examine such an impact, there was some sense that searching for and using optimal approaches became a norm of the class. This unexplored outcome could be an important benefit of promoting flexibility. 


\section{References}

Baroody, A. J., \& Dowker, A. (Eds.). (2003). The development of arithmetic concepts and skills: Constructing adaptive expertise. Mahwah, NJ: Lawrence Erlbaum.

Baroody, A. J., Feil, Y., \& Johnson, A. (2007). An alternative reconceptualization of procedural and conceptual knowledge. Journal for Research in Mathematics Education, 38(2), 115131.

Baxter, J., Woodward, J., Voorhies, J., \& Wong, J. (2002). We talk about it, but do they get it? Learning Disabilities Research \& Practice, 17(3), 173-185.

Blinded (2008).

Blinded (2009).

Blöte, A. W., Klein, A. S., \& Beishuizen, M. (2000). Mental computation and conceptual understanding. Learning and Instruction, 10, 221-247.

Blöte, A. W., Van der Burg, E., \& Klein, A. S. (2001). Students' flexibility in solving two-digit addition and subtraction problems: Instruction effects. Journal of Educational Psychology, 93, 627-638.

Byrnes, J. P., \& Wasik, B. A. (1991). Role of conceptual knowledge in mathematical procedural learning. Developmental Psychology, 5, 777-786.

Cuoco, A. Goldenberg, E. P., Mark, J. (1996). Habits of mind: An organizing principle for mathematics curricula. Journal of Mathematical Behavior, 15, 375-402.

Hatano, G., \& Inagaki, K. (1986). Two courses of expertise. In H. Stevenson, H. Azuma \& K. Hakuta (Eds.), Child development and education in Japan (pp. 262-272). New York: W.H. Freeman and Company. 
Hiebert, J. (1986). Conceptual and procedural knowledge: The case of mathematics. Hillsdale, NJ: Lawrence Erlbaum.

Klein, A. S., Beishuizen, M., \& Treffers, A. (1998). The empty number line in Dutch second grades: Realistic versus gradual program design. Journal for Research in Mathematics Education, 29(4), 443-464.

Krutetskii, V. A. (1976). The psychology of mathematical abilities in school children (J. Teller, Trans.). Chicago: University of Chicago Press.

Lemaire, P., \& Siegler, R. S. (1995). Four aspects of strategic change: Contributions to children's learning of multiplication. Journal of Experimental Psychology: General, 124, 83-97.

Luchins, A. S. (1942). Mechanization in problem solving. Psychological Monographs, 54(248).

Miller, P. H., \& Seier, W. L. (1994). Strategy utilization deficiencies in children: When, where, and why. In H. W. Reese (Ed.), Advances in child development and behavior (Vol. 25, pp. 107-156). New York, NY: Academic Press.

National Council of Teachers of Mathematics. (2006). Curriculum Focal Points for prekindergarten through grade 8 mathematics: A quest for coherence. Reston, VA: Author.

National Mathematics Advisory Panel. (2008). Foundations for success: The final report of the National Mathematics Advisory Panel. Washington, DC: U.S. Department of Education. National Research Council. (2001). Adding it up: Helping children learn mathematics. Washington, DC: National Academy Press.

Richland, L. E., Zur, O., \& Holyoak, K. J. (2007). Cognitive supports for analogies in the mathematics classroom. Science, 316, 1128-1129. 
Rittle-Johnson, B., Siegler, R. S., \& Alibali, M. W. (2001). Developing conceptual understanding and procedural skill in mathematics: An iterative process. Journal of Educational Psychology, 93(2), 346-362.

Rittle-Johnson, B., \& Star, J. R. (2007). Does comparing solution methods facilitate conceptual and procedural knowledge? An experimental study on learning to solve equations. Journal of Educational Psychology, 99(3), 561-574.

Rittle-Johnson, B, \& Star, J.R. (2009). Compared to what? The effects of different comparisons on conceptual knowledge and procedural flexibility for equation solving. Journal of Educational Psychology, 101(3), 529-544.

Rittle-Johnson, B., Star, J.R., \& Durkin, K. (2009). The importance of familiarity when comparing examples: Impact on conceptual and procedural knowledge of equation solving. Journal of Educational Psychology, 101(4), 836-852.

Schwartz, D. L., \& Bransford, J. D. (1998). A time for telling. Cognition and Instruction, 16(4), 475-522.

Siegler, R. S. (1996). Emerging minds: The process of change in children's thinking. New York: Oxford University Press.

Star, J. R. (2005). Reconceptualizing procedural knowledge. Journal for Research in Mathematics Education, 36(5), 404-411.

Star, J. R. (2007). Foregrounding procedural knowledge. Journal for Research in Mathematics Education, 38(2), 132-135.

Star, J. R., \& Rittle-Johnson, B. (2008). Flexibility in problem solving: The case of equation solving. Learning and Instruction, 18, 565-579. 
Star, J.R., \& Rittle-Johnson, B. (2009). It pays to compare: An experimental study on computational estimation. Journal of Experimental Child Psychology, 102, 408-426.

Star, J. R., \& Seifert, C. (2006). The development of flexibility in equation solving. Contemporary Educational Psychology, 31(280-300).

Sweller, J., van Merrienboer, J. J. G., \& Paas, F. G. W. C. (1998). Cognitive architecture and instructional design. Educational Psychology Review, 10(3), 251-296.

Torbeyns, J., Verschaffel, L., \& Ghesquiere, P. (2006). The development of children's adaptive expertise in the number domain 20 to 100. Cognition and Instruction, 24(4), 439-465.

Verschaffel, L., Luwel, K., Torbeyns, J., \& Van Dooren, W. (2007). Developing adaptive expertise: A feasible and valuable goal for (elementary) mathematics education? Ciencias Psicologicas, 2007(1), 27-35.

Wertheimer, M. (1959). Productive thinking (Enlarged ed.). New York: Harper \& Brothers.

Woodward, J., \& Baxter, J. (1997). The effects of an innovative approach to mathematics on academically low-achieving students in inclusive settings. Exceptional Children, 63(3), 373-388.

Woodward, J., \& Montague, M. (2002). Meeting the challenge of mathematics reform for students with LD. The Journal of Special Education, 36(2), 89-101.

Wu, H. H. (1999). Basic skills versus conceptual understanding: A bogus dichotomy in mathematics education. American Educator, 23, 14-19, 50-52. 


\section{Footnotes}

${ }^{1}$ In this paper, problems refers to routine algebra exercises rather than word problems or other non-routine tasks. 
Table 1

Sample Side-by-Side Format for Comparing and Contrasting Methods

\begin{tabular}{|c|c|}
\hline $\begin{array}{r}4(x+1)=19 \\
4 x+4=19 \\
4 x=15 \\
x=\frac{15}{4}\end{array}$ & $\begin{array}{r}4(x+1)=19 \\
x+1=\frac{19}{4} \\
x=\frac{15}{4}\end{array}$ \\
\hline $\begin{array}{r}3(x+1)=15 \\
3 x+3=15 \\
3 x=12 \\
x=4\end{array}$ & $\begin{array}{r}3(x+1)=15 \\
x+1=5 \\
x=4\end{array}$ \\
\hline $\begin{array}{r}\frac{2}{3}(x+5)=8 \\
\frac{2}{3} x+\frac{10}{3}=8 \\
\frac{2}{3} x=\frac{14}{3} \\
x=7\end{array}$ & $\begin{aligned} \frac{2}{3}(x+5) & =8 \\
x+5 & =12 \\
x & =7\end{aligned}$ \\
\hline
\end{tabular}


Table 2

Sample Problem Types and Strategies Covered

\begin{tabular}{|c|c|c|}
\hline Topic & Sample problem and strategy A & Sample problem and strategy B \\
\hline $\begin{array}{l}\text { Solving linear } \\
\text { equations }\end{array}$ & $\begin{array}{c}\text { Divide first } \\
\begin{array}{c}4(r+13)=20 \\
r+13=5 \\
r=-8\end{array}\end{array}$ & $\begin{array}{c}\text { Distribute first } \\
\begin{array}{c}4(r+13)=20 \\
4 r+52=20 \\
4 r=-32 \\
r=-8\end{array}\end{array}$ \\
\hline $\begin{array}{l}\text { Solving systems of } \\
\text { equations }\end{array}$ & 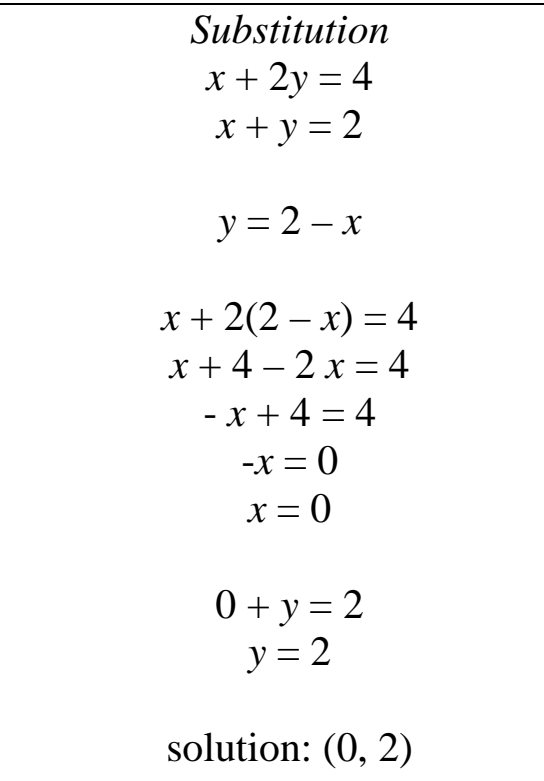 & $\begin{array}{c}\begin{array}{c}\text { Elimination } \\
x+2 y=4 \\
-(x+y=2) \\
0+y=2 \\
y=2 \\
x+2(2)=4 \\
x+4=4 \\
x=0\end{array} \\
\text { solution: }(0,2)\end{array}$ \\
\hline $\begin{array}{l}\text { Simplifying } \\
\text { expressions with } \\
\text { exponents }\end{array}$ & $\begin{array}{c}\text { Expand terms } \\
\left(m^{5}\right)^{2} \\
m^{5} \cdot m^{5} \\
m \cdot m \cdot m \cdot m \cdot m \cdot m \cdot m \cdot m \cdot m \cdot m \\
m^{10}\end{array}$ & $\begin{array}{c}\text { Multiply exponents } \\
\left(m^{5}\right)^{2} \\
m^{(5 \cdot 2)} \\
m^{10}\end{array}$ \\
\hline $\begin{array}{l}\text { Simplifying radical } \\
\text { expressions }\end{array}$ & $\begin{array}{l}\text { Square root first } \\
\qquad \sqrt{\frac{18}{2}} \\
\frac{\sqrt{18}}{\sqrt{2}} \\
\frac{\sqrt{9} \cdot \sqrt{2}}{\sqrt{2}}\end{array}$ & $\begin{array}{c}\text { Simplify first } \\
\sqrt{\frac{18}{2}} \\
\sqrt{9} \\
3\end{array}$ \\
\hline
\end{tabular}




\begin{tabular}{|c|c|c|}
\hline & $\begin{array}{c}\sqrt{9} \\
3\end{array}$ & \\
\hline $\begin{array}{l}\text { Solving quadratic } \\
\text { equations }\end{array}$ & $\begin{array}{c}\text { Square root method } \\
\begin{array}{c}d^{2}-32=16 \\
d^{2}=48 \\
d=\sqrt{48} \\
d= \pm 4 \sqrt{3}\end{array}\end{array}$ & 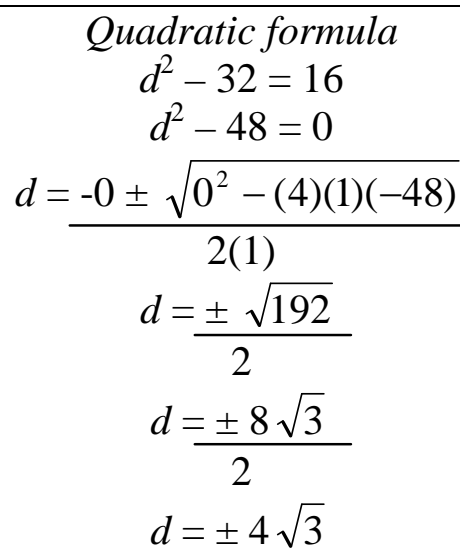 \\
\hline
\end{tabular}

Note: Other strategies for the problem types above were included in the course. For example, factoring was discussed as a method for solving some quadratic equations. In addition, multiple strategies were also discussed for topics not listed above, such as for graphing linear equations. 
Table 3

Percent Correct Scores for Pretest and Posttest

\begin{tabular}{|l|l|l|}
\hline & Pretest & Posttest \\
\hline Xavier & 28 & 80 \\
\hline Nicole & 33 & 54 \\
\hline Annemarie & 33 & 70 \\
\hline Ricardo & 36 & 88 \\
\hline Naomi & 40 & 89 \\
\hline Yvonne & 56 & 88 \\
\hline
\end{tabular}




\section{Figure Captions}

Figure 1. Naomi, Pretest \#28

Figure 2. Naomi, Posttest \#28

Figure 3. Xavier, Test 2 \#2

Figure 4. Annemarie, Quiz 2 \#5 


$$
\text { 28. } \begin{aligned}
\frac{2}{3} w+3 & =10 \\
2 w+9 & =30 \\
2 w & =21 \\
w & =10.5
\end{aligned}
$$




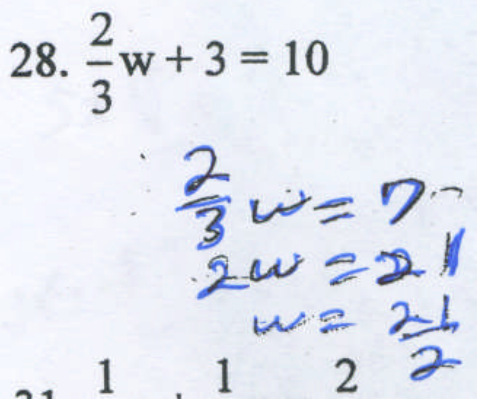


2. $d^{2} \cdot d^{9} \cdot d^{5}$

d.d.d.eld.d.dedd.dd.d

ddd.d.d.d =

$d^{16}$ 
5. $\left(\frac{4 x^{6} y z^{7}}{4 x^{4} z^{5}}\right)^{4}$



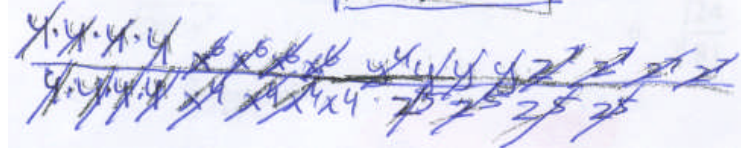

